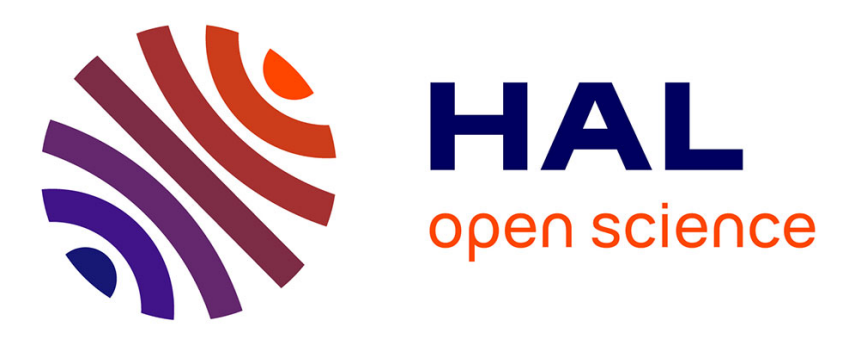

\title{
The effect of silicon impurities on the phase diagram of iron and possible implications for the Earth's core structure
}

\author{
Alexander S. Côté, Lidunka Vočadlo, John P. Brodholt
}

\section{- To cite this version:}

Alexander S. Côté, Lidunka Vočadlo, John P. Brodholt. The effect of silicon impurities on the phase diagram of iron and possible implications for the Earth's core structure. Journal of Physics and Chemistry of Solids, 2009, 69 (9), pp.2177. 10.1016/j.jpcs.2008.03.031 . hal-00565438

\author{
HAL Id: hal-00565438 \\ https://hal.science/hal-00565438
}

Submitted on 13 Feb 2011

HAL is a multi-disciplinary open access archive for the deposit and dissemination of scientific research documents, whether they are published or not. The documents may come from teaching and research institutions in France or abroad, or from public or private research centers.
L'archive ouverte pluridisciplinaire HAL, est destinée au dépôt et à la diffusion de documents scientifiques de niveau recherche, publiés ou non, émanant des établissements d'enseignement et de recherche français ou étrangers, des laboratoires publics ou privés. 


\section{Author's Accepted Manuscript}

The effect of silicon impurities on the phase diagram of iron and possible implications for the Earth's core structure

Alexander S. Côté, Lidunka Vočadlo, John P. Brodholt

PII: S0022-3697(08)00098-X

DOI: doi:10.1016/j.jpcs.2008.03.031

Reference: PCS 5429

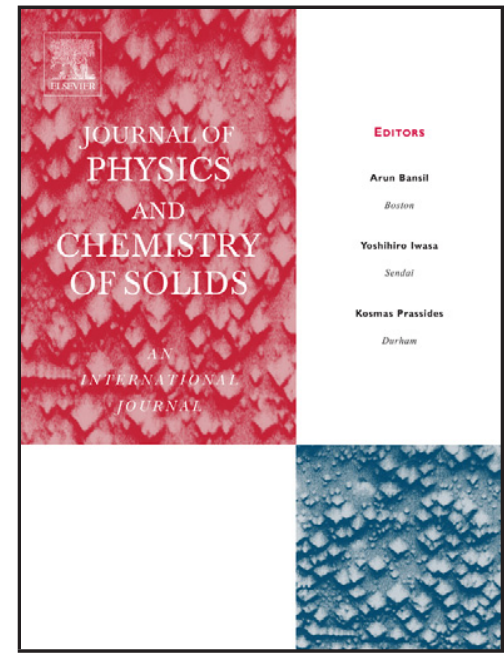

www.elsevier.com/locate/jpcs

To appear in: $\quad$ Journal of Physics and Chemistry of Solids

Cite this article as: Alexander S. Côté, Lidunka Vočadlo and John P. Brodholt, The effect of silicon impurities on the phase diagram of iron and possible implications for the Earth's core structure, Journal of Physics and Chemistry of Solids (2008), doi:10.1016/j.jpcs.2008.03.031

This is a PDF file of an unedited manuscript that has been accepted for publication. As a service to our customers we are providing this early version of the manuscript. The manuscript will undergo copyediting, typesetting, and review of the resulting galley proof before it is published in its final citable form. Please note that during the production process errors may be discovered which could affect the content, and all legal disclaimers that apply to the journal pertain. 


\title{
The effect of silicon impurities on the phase diagram of iron and possible implications for the Earth's core structure
}

\author{
Alexander S. Côté *, Lidunka Vočadlo and John P. Brodholt \\ Department of Earth Sciences, University College London \\ Gower Street, London, WC1E 6BT, United Kingdom
}

\begin{abstract}
The hexagonal close-packed structure (hcp) is the accepted stable form of pure iron (Fe) under Earth's core conditions. Recently, however, a body-centred-cubic (bcc) phase of iron alloyed with lighter elements has been proposed. At relatively modest conditions, experiments have shown that small amounts of silicon ( $\mathrm{Si}$ ) can stabilise the bcc phase with respect to the hcp phase. This result has motivated our present study examining the effect of silicon on the bcc-hcp phase transition. We have performed ab initio calculations on both phases at zero Kelvin; our results are in good agreement with experiment. Extending our results to core pressures and taking into account previous studies of pure iron at core temperatures, we conclude that the bec phase of iron alloyed with silicon is likely to be the stable crystalline phase in the inner core.
\end{abstract}

Keywords: silicon; iron; ab initio; inner core; light elements

\footnotetext{
* Corresponding author. Tel: +44 (0)20 7679 2425; Fax: +44 (0)20 7679 2685, Email address: a.cote@ucl.ac.uk
} 


\section{Introduction}

The Earth's inner core consists largely of iron. Density measurements suggest also the presence of other low atomic weight elements [1]. Quantifying those light elements and determining their role, however, remains a matter of controversy as present experiments cannot simultaneously reproduce the high pressure and temperature conditions of the core. Under ambient conditions, the iron crystal has a body-centred-cubic (bcc) structure. At pressures above $\sim 15 \mathrm{GPa}$, (at room temperature), it adopts a hexagonal-close-packed (hcp) structure which persists to inner-core pressures. Temperature, however, has been shown to act in the opposite sense to pressure. For instance, Vočadlo et al. [5] used ab initio molecular dynamics (MD) simulations under inner-core pressures and temperatures to show that, while the hcp structure is still marginally more stable, the difference in free energy between the hcp and bcc phases becomes very small. Classical potential MD simulations of Belonoshko et al [6] show an even more pronounced effect of temperature and predict that the bcc phase of Fe actually becomes more stable than the hcp structure in the inner-core. Regardless of which is correct, it appears that the free energies of the bcc and hcp phases under core conditions are very similar. The preference of small concentrations of light elements for either phase may thus be the defining factor in determining which Fe structure is stable in the inner core. In particular, it has been shown theoretically [2] and experimentally [3] that, at high pressures, FeSi stabilises in the $\mathrm{CsCl}$ structure, which has identical atomic coordinates to bcc-Fe, rather than in the hcp structure. This suggests that Si may act to stabilise the bcc phase in the core. Indeed, this is supported by low pressure experiments which have shown that, at low concentrations (7.9 weight \%), Si has a large effect on the stability field of bcc Fe, increasing the 
transition pressure to hcp from 10-15 GPa up to $36 \mathrm{GPa}$ at $300 \mathrm{~K}$ [4]. It is possible, therefore, that with an appropriate concentration of Si and at the high temperatures of the core, the bcc phase could be stabilised with respect to the hep and the formation of a bccstructured Fe-Si alloy would be favoured.

In order to test whether Si can stabilise the bcc phase sufficiently to make it the stable phase in the core, we used ab initio calculations to investigate the effect of silicon concentration on both the bcc/hcp phase transition and the equation of state of Fe-Si up to core pressures. In the next section we describe the simulation techniques used in our calculations and the way we obtained the equations of state for the different impurity concentrations in the different iron crystal structures. We then show how the relative enthalpy curves can be used to deduce the stable structure. We calculate the pressure of the bcc-hcp phase transition and compare it to experimental data. Finally we extend these results to Earth's core conditions, taking previous ab initio calculations [5] further by investigating different impurity concentrations and at different volumes, and we discuss possible implications for the inner core structure. Our conclusions give further validity to those of Vočadlo et al. [5].

\section{Quantum mechanical simulation techniques}

The calculations in this work were carried out using Density Functional Theory (DFT) [7] as implemented in the VASP code [8]. The exchange-correlation energy was represented by the generalised gradient approximation (GGA) [9], which achieves the highest accuracy for transition metals. We made use of the projected augmented wave (PAW) 
method, where the wavefunction combines an all-electron core region with a pseudovalence region. The PAW method was introduced by Blöchl [10], and it combines the accuracy of all-electron methods with the efficiency of pseudopotentials. With a relatively small number of plane waves, it can give results that agree accurately with all electron methods. Indeed, it has been previously shown that the use of this method gives accurate results for iron both under ambient and core conditions [11-15].

Unit cells of pure iron were constructed in the bcc and hcp structures. For the bcc case we used the 2-atom cubic unit cell. For the hcp case, the unit cell used was that of the antiferromagnetic II (afmII) structure which contains 8 atoms (see Figure 1), as this was shown to be the stable hcp phase at low pressures $[16,17]$.

VASP calculates the ground state $(\mathrm{T}=0)$ for each set of ionic positions and the electronic free energy is taken as the quantity to be minimised. Care was taken when choosing the kpoint sampling grid and plane wave cutoff energies in order to have an energy convergence of no more than $0.004 \mathrm{eV} /$ atom. A 14x14x14 grid (equivalent to $84 \mathrm{k}$-points in the irreducible Brillouin zone (IBZ)) was used for bcc-Fe and a 9x9x9 grid (125 kpoints in the IBZ) for hep-Fe, with a cutoff energy of $400 \mathrm{eV}$. The same cutoff energy was used throughout the calculations.

Substitutional defects of silicon were created by replacing Fe with Si in hcp and bcc supercells. Three different concentrations were investigated which bracket the experimental value of $7.9 \mathrm{wt} \%$, namely $\sim 3.2, \sim 6.7$ and $\sim 10.4 \mathrm{wt}$. \%. Specifically, for both structures, that meant a substitutional defect of one, two and three Si atoms respectively 
for each of the above concentrations. The supercell contained 16 atoms both in the bcc (a $2 \times 2 \times 2$ multiplication of the 2-atom bcc unit cell) and in the hep structure (a $1 \times 1 \times 2$ multiplication of the afm II unit cell). A $5 \times 5 \times 5$ k-point grid (18 k-points in the IBZ) was used for the bcc and a 9x9x5 (123 k-points in the IBZ) for the hep phase. The structures were then allowed to relax and the total energy was calculated. In the supercells containing more than one Si defect, a number of trials were performed with the different possible configurations of the defect atoms. In the hcp supercells the Si atoms were placed at symmetric positions as far away from each other as possible, taking into account the periodic boundary conditions. This proved to be the most stable configuration. In the bcc case, two different substitutional configurations were found to be favoured at high and low pressures respectively, so both were taken into account.

Finding the ground state free energy $\mathrm{E}$ for a set of different volumes $\mathrm{V}$ allowed us to fit the E-V data to a $3^{\text {rd }}$ order Birch-Murnaghan equation of state (EOS) [18] of the following form:

$E(V)=\frac{9}{4} K_{0} V_{0} \frac{V_{0}}{V}\left[\frac{1}{2}\left(\frac{V_{0}}{V}\right)^{\frac{1}{3}}-\left(\frac{V}{V_{0}}\right)^{\frac{1}{3}}\right]+\frac{9}{16} K_{0}\left(K^{\prime}-4\right) V_{0}\left(\frac{V_{0}}{V}\right)\left[\left(\frac{V_{0}}{V}\right)-3\left(\frac{V_{0}}{V}\right)^{\frac{1}{3}}+3\left(\frac{V}{V_{0}}\right)^{\frac{1}{3}}\right]+E_{0}-\frac{9}{16} K_{0} V_{0}\left(K^{\prime}-6\right)$

The resulting EOS parameters were then used to obtain the enthalpies $\mathrm{H}$ at those volumes, and to plot the enthalpy as a function of pressure for the bcc and hep phases of the different Si concentrations. 


\section{Results}

The equation of state parameters for each silicon concentration are shown in Table 1. The parameters for pure iron [19-22] are also displayed, where the volume underestimation due to the GGA functional is apparent. For the higher concentrations of $\mathrm{Si}$, where different Si configurations are favoured at low and high pressures, both sets of parameters are displayed. For the $6.7 \%$ concentration, this change of stability between the two configurations in bcc takes place at around $210 \mathrm{GPa}$, whereas for the $10.4 \%$ concentration, it takes place at around $80 \mathrm{GPa}$. The equation of state parameters were used to plot the enthalpy curves as a function of pressure for pure $\mathrm{Fe}$ and for the three different concentrations of Si considered here.

The enthalpy-pressure curves are plotted in Figure 2. Also indicated are the transition pressures for the pseudo-univariant reaction (i.e. the transition from one phase to another with the same composition, ignoring any possible solid-solutions). The calculated transition pressures are $\sim 7 \mathrm{GPa}, \sim 11 \mathrm{GPa}, \sim 16 \mathrm{GPa}$ and $\sim 41 \mathrm{GPa}$, for concentrations of $0 \%, 3.2 \%, 6.7 \%$ and $10.4 \%$ Si respectively. In Figure 3 we plot the change in the transition pressure for the pseudo-univariant reaction as a function of composition. As can be seen, the effect of $\mathrm{Si}$ on the transition pressure is strongly non-linear. Also shown is the pressure range of the room temperature solid solution as found experimentally by Lin et al. [4] for a bulk composition containing 7.9\% Si. Although the experimental solid solution cannot be used to establish the exact pressure of the pseudo-univariant reaction, for a particular composition the pseudo-univariant reaction must be bracketed by the minimum and maximum pressure where both phases are present. The experimental two- 
phase region at that composition starts at about $4 \mathrm{GPa}$ above the pure $\mathrm{Fe}$ transition and persists for about $20 \mathrm{GPa}$ [4]. As can be seen in the figure, we calculate that $7.9 \% \mathrm{Si}$ will increase the pressure of the pseudo-univariant transition by $\sim 16 \mathrm{GPa}$, a value that falls within the experimental solid solution range. This adds some confidence to our results and confirms that the presence of Si can strongly increase the stability of the bcc phase over hep.

We repeated the $0 \mathrm{~K}$ calculations at the pressure of the inner core boundary (ICB) (330 GPa). It is worth noting that the magnetic moments at such high pressures greatly diminish in the bcc phase, and disappear completely in the hep phase. The results are given in Table 2. For pure iron, the calculated difference in enthalpies between the bcc and hcp structures of pure iron is $660 \mathrm{meV}$ in favour of the hcp structure. As with the low pressure results, we find that Si strongly prefers the bcc phase, and in fact, at $10.4 \mathrm{wt} \%$, the enthalpy difference has dropped to only $115 \mathrm{meV}$. We also find, again consistent with the low pressure results, that the enthalpy difference drops almost linearly with increasing concentration of $\mathrm{Si}$. These results show that at $330 \mathrm{GPa}$, Si defects are about $2.5 \mathrm{eV}$ per defect atom more stable in the bcc structure than in the hcp phase. This means that, for example, the suggested $\sim 5 \mathrm{~mol} \%$ concentration of $\mathrm{Si}$ in the inner core $[4,5,23]$ would lower the enthalpy difference between the two phases by $125 \mathrm{meV}$.

It is known that bcc-Fe is dynamically unstable at $0 \mathrm{~K}$ and high pressures [24, 25]. Our results, however, show that the addition of Si decreases the free energy difference between the two phases; the implication of this is that the magnitude of the instability is 
also reduced. Indeed it may even be the case that the incorporation of Si removes the instability altogether, even at $0 \mathrm{~K}$.

\section{Discussion and Conclusion}

Even though there is no doubt from our results that the inclusion of Si tends to stabilise bcc-Fe, the calculated enthalpy differences between the two pure Fe structures at $\mathrm{T}=0$ still favour hcp stability at inner core pressures, at least for the concentrations considered here. However, the high temperatures of the core alter this significantly and recent ab initio results show that the difference between the free energies of the two phases of pure iron at ICB pressures and temperatures is only $\sim 33 \mathrm{meV}$ [5]. Since, as shown above, Si stabilises bcc over hcp by $2.5 \mathrm{eV}$ per defect atom, less than $1.5 \mathrm{~mol} \% \mathrm{Si}$ is needed to overcome the $33 \mathrm{meV}$ required to make bcc stable. The higher but still modest concentration of 5 mol.\% would provide a stabilisation enthalpy of $125 \mathrm{meV}$. This shows that even the relatively small concentrations of Si suggested for the inner core [26] are more than enough to stabilise bcc-Fe in the inner core. We conclude, therefore, that the presence of the bcc phase in the inner core cannot be ruled out.

\section{Acknowledgements}

The work of ASC was supported by NERC grant NE/C519662/1. We would also like to thank the Royal Society for the University Fellowship awarded to LV. 


\section{References}

[1] F. Birch, Elasticity and Constitution of the Earths Interior, Transactions of the New York Academy of Sciences, 14, (1951), 72-76.

[2] L. Vočadlo, et al., Crystal Structure, Compressibility and Possible Phase Transitions in Epsilon-Fesi Studied by First-Principles Pseudopotential Calculations, Acta Crystallogr. Sect. B-Struct. Sci., 55, (1999), 484-493.

[3] D.P. Dobson, et al., A New High-Pressure Phase of Fesi, Am. Miner., 87, (2002), 784-787.

[4] J.F. Lin, et al., Iron-Silicon Alloy in Earth's Core?, Science, 295, (2002), 313-315.

[5] L. Vočadlo, et al., Possible Thermal and Chemical Stabilization of Body-CentredCubic Iron in the Earth's Core, Nature, 424, (2003), 536-539.

[6] A.B. Belonoshko, et al., Stability of the Body-Centred-Cubic Phase of Iron in the Earth's Inner Core, Nature, 424, (2003), 1032-1034.

[7] P. Hohenberg and W. Kohn, Inhomogeneous Electron Gas, Physical Review B, 136, (1964), B864.

[8] G. Kresse and J. Furthmuller, Efficient Iterative Schemes for Ab Initio Total-Energy Calculations Using a Plane-Wave Basis Set, Physical Review B, 54, (1996), 1116911186.

[9] Y. Wang and J.P. Perdew, Correlation Hole of the Spin-Polarized Electron-Gas, with Exact Small-Wave-Vector and High-Density Scaling, Physical Review B, 44, (1991), 13298-13307.

[10] P.E. Blöchl, Projector Augmented-Wave Method, Physical Review B, 50, (1994), 17953-17979.

[11] H.K. Mao, et al., Phonon Density of States of Iron up to 153 Gigapascals, Science, 292, (2001), 914-916.

[12] L. Vočadlo, et al., The Properties of Iron under Core Conditions from First Principles Calculations, Physics of the Earth and Planetary Interiors, 140, (2003), 101125.

[13] D. Alfè, et al., Thermodynamics of Hexagonal-Close-Packed Iron under Earth's Core Conditions, Physical Review B, 6404, (2001), 16. 
[14] L. Vočadlo, et al., First Principles Calculations on Crystalline and Liquid Iron at Earth's Core Conditions, Faraday Discuss., (1997), 205-217.

[15] G.D. Price, et al., in: R.S.J. Sparks and C.J. Hawksworth (Eds), The Earth's Core: An Approach from First Principles. Geophys. Monogr. Ser.: The State of the Planet: Frontiers and Challenges in Geophysics, 2004, 1-12

[16] G. Steinle-Neumann, et al., Magnetism in Dense Hexagonal Iron, Proceedings of the National Academy of Sciences of the United States of America, 101, (2004), 33-36.

[17] L. Vočadlo, et al., An Ab Initio Study of Nickel Substitution into Iron, Earth and Planetary Science Letters, 248, (2006), 147-152.

[18] J.P. Poirier, Introduction to the Physics of the Earth's Interior, Cambridge University Press, Cambridge, U.K., 1991, 66-74

[19] M.W. Guinan and D.N. Beshers, Pressure Derivatives of Elastic Constants of Alpha-Iron to $10 \mathrm{Kbs}$, Journal of Physics and Chemistry of Solids, 29, (1968), 541-\&.

[20] A. Dewaele, et al., Quasihydrostatic Equation of State of Iron above 2 Mbar, Physical Review Letters, 97, (2006), 4.

[21] H.K. Mao, et al., Static Compression of Iron to $300 \mathrm{Gpa}$ and Fe0.8ni0.2 Alloy to $260 \mathrm{Gpa}$ - Implications for Composition of the Core, Journal of Geophysical ResearchSolid Earth and Planets, 95, (1990), 21737-21742.

[22] G. Steinle-Neumann, et al., First-Principles Elastic Constants for the Hcp Transition Metals Fe, Co, and Re at High Pressure, Physical Review B, 60, (1999), 791-799.

[23] L. Stixrude, et al., Composition and Temperature of Earth's Inner Core, Journal of Geophysical Research-Solid Earth, 102, (1997), 24729-24739.

[24] L. Stixrude and R.E. Cohen, Constraints on the Crystalline Structure of the Inner Core: Mechanical instability of BCC iron at high pressure, Geophysical Research Letters, 22, (1995), 125-128.

[25] P. Söderlind, et al., First-Principles Theory of Iron up to Earth-core Pressures: Structural, Vibrational and Elastic Properties, Physical Review B, 53, (1996), 1406414072.

[26] C.J. Allègre, Chemical Composition of the Earth and Constraints on Its Early History and Chronology, Meteoritics, 29, (1994), 437-437. 


\section{Figure and table captions}

Figure 1: The afmII structure. The arrows on the atoms represent the spin direction. White atoms are positioned at $\mathrm{z}=1 / 4$, black atoms at $\mathrm{z}=3 / 4$. The 8 -atom cell that was used is marked with dashed lines. It is the smallest possible cell for this structure, and is a $2 \times 2 \times 1$ multiplication of the primitive hep cell.

Figure 2: Calculated enthalpy curves for bcc and hcp structures at 3 different concentrations of silicon. The arrows indicate the transition pressures for the pseudounivariant reactions.

Figure 3: Change in the transition pressure $\left(\Delta \mathrm{P}=\mathrm{P}_{\mathrm{tr}}{ }^{\mathrm{FeX}}-\mathrm{P}_{\mathrm{tr}}{ }^{\mathrm{Fe}}\right)$ for the pseudo-univariant reaction as a function of composition. The gray bar represents the experimental pressure range of the $300 \mathrm{~K}$ solid solution [4] for a bulk composition containing $7.9 \% \mathrm{Si}$. We can see that our result on the pseudo-univariant reaction at $7.9 \% \mathrm{Si}$ falls well within that range. The solid line is a weighted fit through the ab initio results.

Table 1: The parameters of the Birch-Murnaghan $3^{\text {rd }}$ order equation of state, for the different concentrations of silicon. For pure iron, the experimental parameters are also displayed $(b, d)$.

Table 2: The enthalpy differences between the bcc and hep structures at $\mathrm{P}=330 \mathrm{GPa}$ and $\mathrm{T}=0 \mathrm{~K}$. At $\mathrm{T}=0 \mathrm{~K}$ the hep phase is always more stable, but one can see that the difference drops almost linearly as the percentage of Si increases. 


\section{Tables}

\begin{tabular}{|l|l|l|l|l|}
\hline wt. \%Si & Fe structure & $\mathbf{V}_{\mathbf{0}}\left(\AA^{\mathbf{3}}\right)$ & $\mathbf{K}_{\mathbf{0}}(\mathbf{G P a})$ & $\mathbf{K}$ \\
\hline \multirow{4}{*}{$\mathbf{0}$} & bcc & $11.39(1)$ & $203(3)$ & $4.36(3)$ \\
& ref. [17] & $11.355(7)$ & $201.502(8)$ & $4.29(1)$ \\
& ref. [19] & 11.78 & 166.4 & 5.29 \\
\cline { 2 - 5 } & hcp-afmII & $10.48(3)$ & $229(7)$ & $5.12(13)$ \\
& ref. [22] & 10.55 & 209 & 5.2 \\
& ref. [21] & $11.176(17)$ & $165(4)$ & $5.33(90)$ \\
\hline \multirow{3}{*3.2}{} & bcc & $11.34(2)$ & $201(3)$ & $4.39(3)$ \\
\cline { 2 - 5 } & hcp-afmII & $10.55(8)$ & $216(12)$ & $4.95(19)$ \\
\hline \multirow{3}{*6.7}{} & bcc 1: & $11.15(3)$ & $216(6)$ & $4.35(8)$ \\
& bcc 2: & $11.28(3)$ & $198(6)$ & $4.41(9)$ \\
\cline { 2 - 5 } & hcp-afmII & $10.55(4)$ & $238(9)$ & $4.84(14)$ \\
\hline \multirow{3}{*}{$\sim \mathbf{1 0 . 4}$} & bcc 1: & $11.12(4)$ & $212(7)$ & $4.47(9)$ \\
& bcc 2: & $11.31(5)$ & $156(7)$ & $5.25(12)$ \\
\cline { 2 - 5 } & hcp-afmII & $10.61(3)$ & $239(8)$ & $4.67(11)$ \\
\hline
\end{tabular}

1: Derived from the Si configuration most stable at low pressures.

2: Derived from the Si configuration most stable at core pressures.

Table 1: The parameters of the Birch-Murnaghan $3^{\text {rd }}$ order equation of state, for the different concentrations of silicon. For pure iron, the experimental parameters are also displayed $(b, d)$. 


\begin{tabular}{|c|c|}
\hline wt. \% Si & $\mathbf{\Delta H}=\mathbf{H}_{\text {bcc }}-\mathbf{H}_{\text {hcp }}$ \\
\hline 0 & 660 \\
\hline 3.2 & 503 \\
\hline 6.7 & 354 \\
\hline 10.4 & 115 \\
\hline
\end{tabular}

Table 2: The enthalpy differences between the bcc and hcp structures at $P=330 G P a$ and $T=0 K$. At $T=0 K$ the hcp phase is always more stable, but one can see that the difference drops almost linearly as the percentage of Si increases. 


\section{Figures}

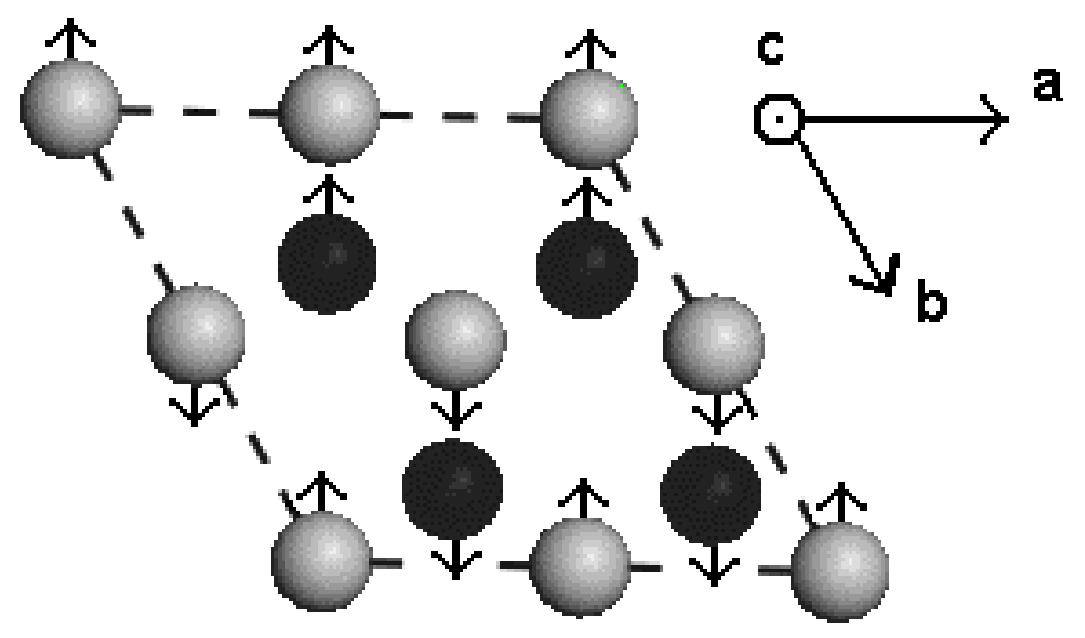

Figure 1: The afmII structure. The arrows on the atoms represent the spin direction. White atoms are positioned at $z=1 / 4$, black atoms at $z=3 / 4$. The 8-atom cell that was used is marked with dashed lines. It is the smallest possible cell for this structure, and is a $2 \times 2 \times 1$ multiplication of the primitive hcp cell. 

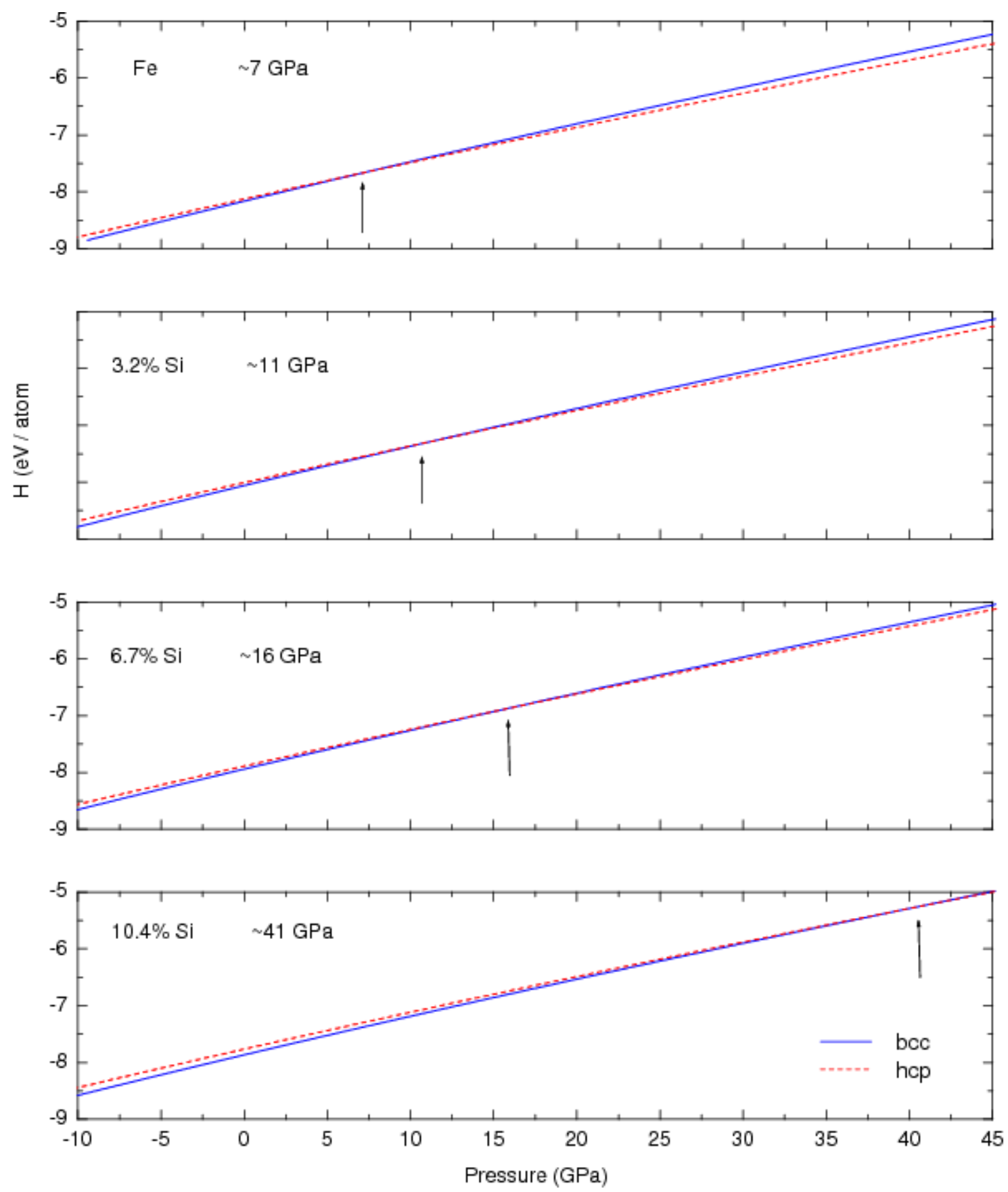

Figure 2: Calculated enthalpy curves for bcc and hcp structures at 3 different concentrations of silicon. The arrows indicate the transition pressures for the pseudounivariant reactions. 


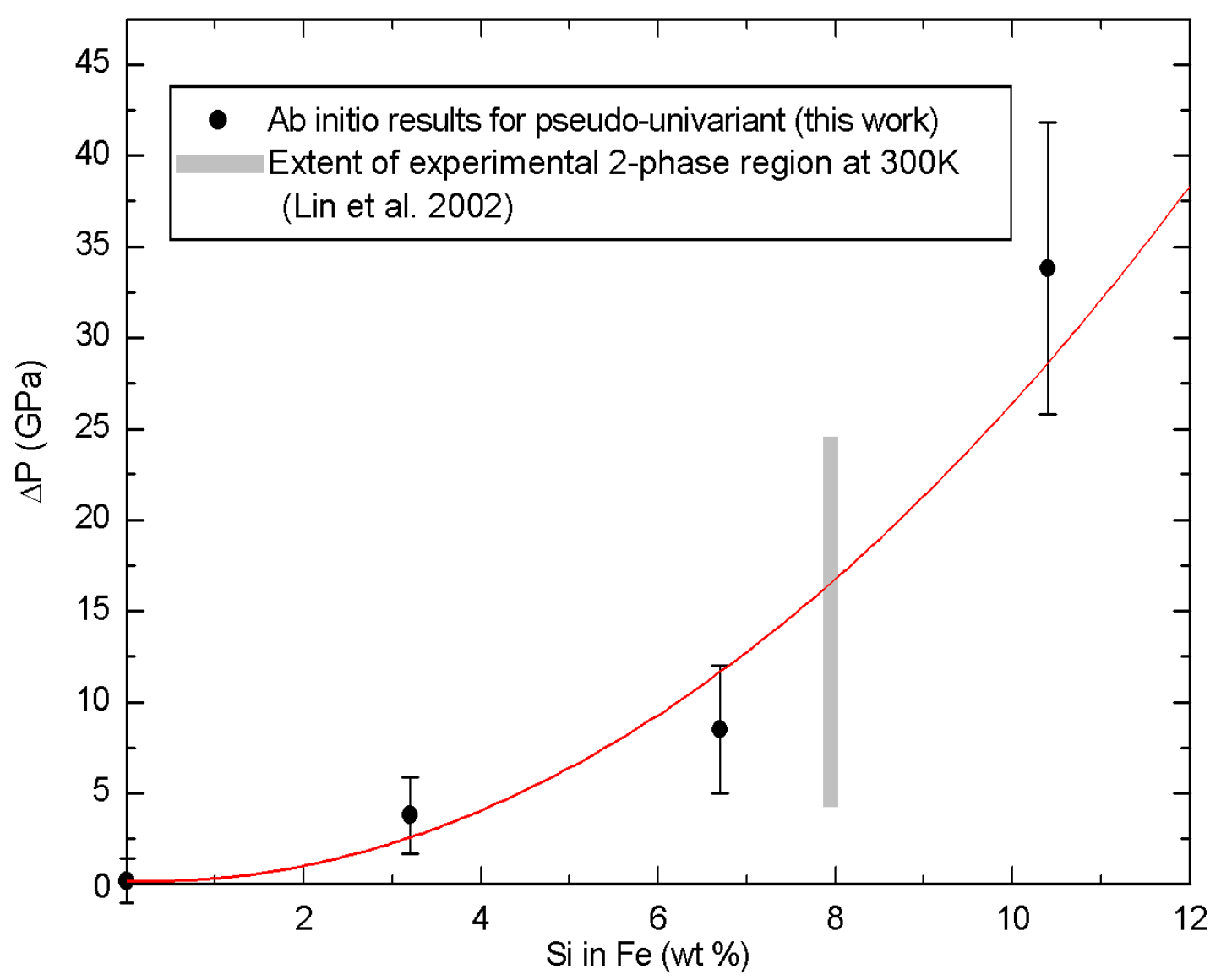

Figure 3: Change in the transition pressure $\left(\triangle P=P_{t r}{ }^{F e X}-P_{t r}{ }^{F e}\right)$ for the pseudo-univariant reaction as a function of composition. The gray bar represents the experimental pressure range of the $300 \mathrm{~K}$ solid solution [4] for a bulk composition containing 7.9\% Si. We can see that our result on the pseudo-univariant reaction at $7.9 \%$ Si falls well within that range. The solid line is just a weighted fit through the ab initio results. 